\title{
A Method for Detecting Small Vibrations of Particles by LDV for Particle Size and Electrostatic Charge Measurements $\dagger$
}

\author{
Yoji Nakajima \\ Dept. of Chem. Process Eng., Hokkaido University* \\ Takashi Sato \\ Dept. of Electrical Eng., Hokkaido Institute of Technology**
}

\begin{abstract}
A mathematical model based on an ideal LDV (Laser Doppler Velocimeter) signal indicates that the phase and amplitude of a light scattering particle vibrating sinusoidally within the interference fringes can be obtained from the Fourier integrals of the signal. Although actual LDV signals are distorted, it is found empirically that the use of a Hanning window is very effective for the removal of the undesirable effect of distortion. Hence the size and electrostatic charge of a particle being excited in an AC electric field can be calculated using the Fourier analysis of the LDV signal. A computer simulation shows that the phase can be determined very accurately, say, within $0.1^{\circ}$ error if so desired. The lower limit of the amplitude measurement is ca. $0.2 \mu \mathrm{m}$ within a few percent relative error. Therefore the present method is expected to provide a convenient device for measuring the size and the charge of particles of around $1 \mu \mathrm{m}$ in diameter.
\end{abstract}

\section{Introduction}

Electrostatic and electrodynamic phenomena will hopefully be applicable to powder handling processes such as for classification, sorting, conveying, dispersion, mixing, coating, and so on. The most important properties of powder particles for such applications are of course the charge and the size. Various methods have been used for the measurement of the electrostatic charge of powders, however, most of them are not applicable to the measurement of the charge of single particles. Recently, a few devices used for simultaneous measurements of the charge and size of individual particles have been proposed $^{1)}$, some of which are commercially available for practical use ${ }^{2)}$.

In a previous paper ${ }^{3}$, the authors proposed

\footnotetext{
* N13 W8, Kita-ku, Sapporo 060 JAPAN TEL. (011) 7162111

** 7-Jo 15-Chome, Teine-ku, Sapporo 006 JAPAN TEL. (011) 6812161

$\dagger$ This report was originally printed in Funsai No.35, 11-20 (1991) in Japanese, before being translated into English with the permission of Hosokawa Micron Corporation, Japan.
}

one such device in which the waveform of a LDV signal emitted by a vibrating particle in the LDV fringes was geometrically analyzed to obtain the phase and amplitude of the particle vibration. Since the vibration of the charged particle in the device was induced by a controlled $\mathrm{AC}$ electric field, the phase and amplitude were converted into the size and charge of the particle. The accuracy of the measurement obtained with the device was satisfactory when the amplitude of the particle vibration was larger than the LDV fringe spacing. However, it becomes rather difficult to attain a sufficiently large amplitude with a moderate $\mathrm{AC}$ field when the device is applied to the measurements of aerosol particles usually encountered in the atmosphere.

In the present paper, a new method for analyzing LDV signals is proposed, with which particle amplitudes as small as one tenth the fringe size can be measured.

\section{Theoretical background}

\subsection{Basic principle of size and charge mea- surements}

Airborne particles with electrostatic charge 
flow through the measuring space between a pair of parallel plate electrodes, to which an $\mathrm{AC}$ high voltage having an angular frequency of $\omega$ is applied. As shown in Fig. 1, the particle is forced to vibrate in the $\mathrm{x}$ direction with an amplitude $\mathrm{A}$ under the effect of an $\mathrm{AC}$ electric field, $\mathrm{E} \cdot \sin (\omega \mathrm{t})$.

Let the displacement of a vibrating particle in the measuring space be

$\mathrm{x}=\mathrm{A} \sin (\omega \mathrm{t}-\phi)+\mathrm{x}_{0}$

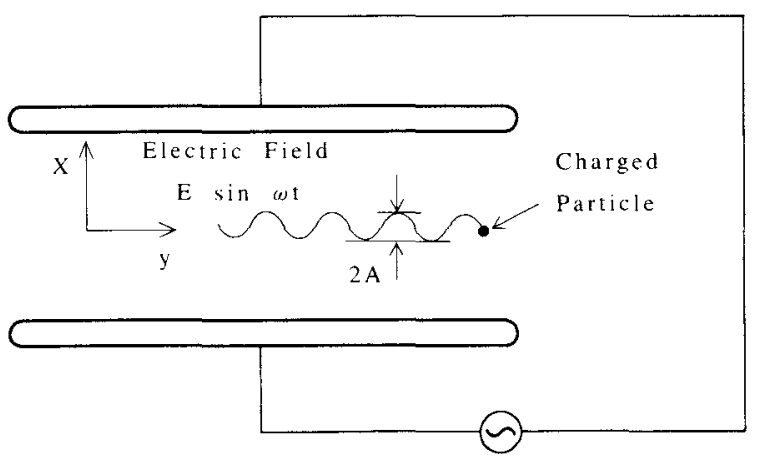

Fig. 1 AC electric field exerting on charged particle

As will be described later, the amplitude $A$ and the phase lag $\phi$ can be obtained from the Fourier analysis of the LDV signal. The parameters of the vibration, $A$ and $\phi$ are related to the following two parameters, which are characteristic to the particle, i.e., the electric mobility $Z$ and the relaxation time $\tau$.

$\mathrm{A}=\frac{\mathrm{ZE}}{\omega \sqrt{(\tau \omega)^{2}+1}}=\frac{Z \mathrm{E}}{\omega} \sin \phi$

$\phi=\frac{\pi}{2}+\tan ^{-1}(\tau \omega)$

We may assume a negative sign for $\mathrm{Z}$ and $\mathrm{A}$ if the particle charge is negative. Note that the phase lag $\phi$ is the phase difference between the applied AC electric field and the particle displacement (and not the particle velocity), and hence it is an angle between $90^{\circ}$ and $180^{\circ}$.

The ratio of the two parameters, $\frac{Z}{\tau}$, gives the specific charge of the particle, namely, the charge per unit mass of the particle. Further, if the particle density is known, the particle diameter $D_{p}$ and the charge $q$ can be calculated from these parameters as follows:
$\mathrm{D}_{\mathrm{p}}=\sqrt{\frac{18 \mu \tau}{\mathrm{C} \rho_{\mathrm{p}}}}$

$\mathrm{q}=\frac{3 \pi \mu \mathrm{D}_{\mathrm{p}} \mathrm{Z}}{\mathrm{C}}$

where $\mu$ is the viscosity of the surrounding medium (air), $\rho_{\mathrm{p}}$ is the density of the particle, and $\mathrm{C}$ is the Cunningham correction factor for molecular slip. The correction factor $\mathrm{C}$ is expressed by an empirical equation.

$\mathrm{C}=1+\frac{2 \Lambda\left\{\mathrm{a}_{1}+\mathrm{a}_{2} \exp \left(\frac{-\mathrm{a}_{3} \mathrm{D}_{\mathrm{p}}}{\Lambda}\right)\right\}}{\mathrm{D}_{\mathrm{p}}}$

where $a_{n}$ 's are the empirical constants, $\Lambda$ is the mean free path of the medium; for normal air, $\mathrm{a}_{1}=1.257, \mathrm{a}_{2}=0.400, \mathrm{a}_{3}=0.55$, and $\Lambda=0.065$ $\mu \mathrm{m}$.

Eq. (4) requires an iterative method for obtaining $\mathrm{D}_{\mathrm{p}}$. Starting with $\mathrm{C}=1$, the first approximation for $D_{p}$ is calculated from Eq. (4), then a better value for $\mathrm{C}$ is estimated using Eq. (6). The iteration converges rapidly.

Eqs. (4) and (5) are derived from a steady Stokes' approximation, in which only the viscous term in the Navier-Stokes equation is taken into account. If the inertial term in the Navier-Stokes equation is included in the calculation (the Basset approximation), the corresponding expressions for Eqs. (4) and (5) may be written as follows:

$\mathrm{D}_{\mathrm{p}}=\sqrt{\frac{18 \mu\left\{(1+\sigma) \tau-\frac{\sigma}{\omega}\right\}}{\mathrm{C} \rho_{\mathrm{p}}}}$

$\mathrm{q}=\frac{3 \pi \mu \mathrm{D}_{\mathrm{p}} Z(1+\sigma)}{\mathrm{C}}$

where $\sigma=\mathrm{D}_{\mathrm{p}} \sqrt{\left(\frac{\omega \rho_{\mathrm{g}}}{8 \mu}\right)}$

$\left(\rho_{\mathrm{g}}\right.$, the density of surrounding gas). Our recent experiment, in which the measurement was carried out repeatedly many times with a single test particle, showed that $D_{p}$ calculated from Eq. (4) depended on the vibrating frequency used for the measurement but $D_{p}$ from Eq. (7) remained constant if the amplitude was not too large. Therefore, Eq. (7) is believed to be more accurate for calculating $D_{p}$ than Eq. (4), though the use of the Cunningham correction factor in Eqs. (7) and (8) has not been justified yet. 


\subsection{LDV signal}

As is well-known, the LDV fringes can be moved at a constant velocity using Bragg cells driven at two different frequencies. The LDV signal emitted by a particle in the moving fringes is an $\mathrm{F}-\mathrm{M}$ wave ${ }^{4}$, of which the frequency is shifted from the carrier frequency (i. e., the difference between the drive frequencies of the Bragg cells) by an amount proportional to the particle velocity. Therefore, an F-M wave demodulator for the LDV signal, which tracks the instantaneous frequency of the LDV signal and transmits a continuous velocity signal, may be used for monitoring the velocity of the particle. Mazumder et al. ${ }^{1}$ successfully used such a conventional demodulator to measure the phase lag of a vibrating particle in the moving fringes.

In our opinion, however, conventional $\mathrm{F}-\mathrm{M}$ wave demodulators may not be the best device for the present measurement, because they are not necessarily designed for the particular purpose of measuring the phase and amplitude of the sinusoidal motion of particles. In the present paper, LDV signals emitted by particles in pure sinusoidal motion with respect to the LDV fringes are mathematically examined to develop a more suitable method for the particular purpose of the present measurements. Here the words 'pure sinusoidal motion' also suggest that we will use stationary LDV fringes produced by a simpler optical system without Bragg cells. The beat signal emitted by a particle oscillating within stationary LDV fringes becomes a far different from a usual $\mathrm{F}-\mathrm{M}$ wave. Therefore a digital computer, instead of a demodulator, will be used to analyze LDV signals in the new method.

Based on the diffraction theory, Roberds ${ }^{5}$ has reported a theoretical analysis of an LDV optical system shown in Fig. 2. The photo current or LDV signal, i, can be written as follows:

$$
\begin{aligned}
\mathrm{i} \sim & \exp \left\{-2 \frac{\left(\mathrm{x}^{2}+\mathrm{y}^{2}+\frac{z^{2} \alpha^{2}}{4}\right)}{\mathrm{b}_{0}{ }^{2}}\right. \\
& \times\left\{\cosh \left(\frac{2 \mathrm{xz} \alpha}{\mathrm{b}_{0}{ }^{2}}\right)+\mathrm{V}_{0} \cos (\mathrm{kx})\right\}
\end{aligned}
$$

where $\alpha$ is the intersection angle of the laser beams, $\mathrm{b}_{0}$ the beam waist radius at the intersection, and $\mathrm{k}$ the fringe wave number, i.e., $\frac{2 \pi}{\delta}$. The fringe spacing $\delta$ is expressed for laser

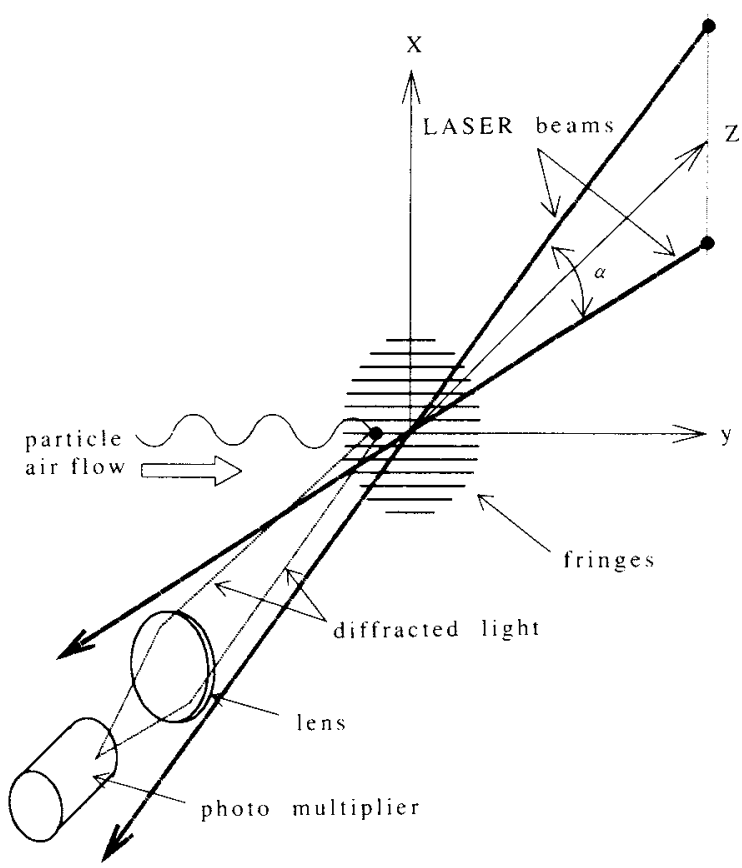

Fig. 2 LDV optical system

beams of wave length $\lambda$ as follows:

$$
\delta=\frac{\lambda}{2 \sin \left(\frac{\alpha}{2}\right)}
$$

Another parameter $\mathrm{V}_{0}$ is a function of the light scattering particle size and optical parameters, but is independent of the coordinates $(x, y, z)$. This parameter is related to the visibility of the LDV signal, i.e., $\mathrm{V}=\frac{\mathrm{V}_{0}}{\cosh \left(\frac{2 \mathrm{xz} \alpha}{\mathrm{b}_{0}{ }^{2}}\right)}$. When the particle size is much smaller than the fringe spacing, $V_{0}$ may be approximated to unity.

For simplicity, we assume an ideal case where the light scattering particle vibrates in the central portion of the LDV fringes with an amplitude much smaller than the beam radius. Then the exponential and the hyperbolic terms in Eq. (9) can be regarded as constants. The beat signal (AC component) may be written as

$$
\mathrm{i}_{\mathrm{b}}=\mathrm{B} \cos \left\{\mathrm{k}\left(\mathrm{A} \sin (\omega \mathrm{t}-\phi)+\mathrm{x}_{0}\right)\right\}
$$

where $\mathrm{B}$ is a constant depending on the optical system and the particle size. As stated in Eq. (2), we assume a negative sign for $\mathrm{A}$ if the particle charge is negative. However, Eq. (11) shows that it is impossible to determine the sign of $\mathrm{A}$; the signal is exactly the same as that when the signs of both $A$ and $x_{0}$ are reversed. Although we may be able to determine the sign if a very 
small velocity component in the $\mathrm{x}$ direction is added to the particle motion ${ }^{3)}$, we confine ourselves to the case where the polarity of the particle charge is known by some other means. In the following, $\mathrm{A}$ is assumed to be positive.

\subsection{Fourier expansion of beat signal}

The bottom curve in Fig. 3 shows a typical example of a beat signal calculated using Eq. (11). The waveform is quite different from normal $\mathrm{F}-\mathrm{M}$ waves. When the amplitude of the particle is comparable to or larger than the fringe spacing, we can find distinctive points denoted by a, b, ... e, at which the direction of the particle motion is reversed. Hence $\phi$ can be determined from the time difference between a peak in the AC field and the nearest reverse point after the peak. Further, the number of peaks in a beat signal between two neighboring reverse points and their positions reflect the relationship between the particle relative location and the phase of oscillation. This information gives the particle amplitude. This is a brief explanation of the method proposed in the previous paper ${ }^{3}$. As may be seen from Eq. (11) and Fig. 3, however, the reverse points in a beat signal become difficult to pinpoint when the amplitude is too small.

Eq. (11) can be expanded ${ }^{6)}$ into an infinite series using Neuman expansion as follows:

$$
\begin{aligned}
\mathrm{i}_{\mathrm{b}} & =\mathrm{B} \cos \left(k \mathrm{x}_{0}\right) \mathrm{J}_{0}(\mathrm{kA}) \\
& -2 \mathrm{~B} \sin \left(k \mathrm{kx}_{0}\right) \sum_{\mathrm{n}=0}^{\infty} \mathrm{J}_{2 \mathrm{n}+1}(\mathrm{kA}) \sin \{(2 \mathrm{n}+1)(\omega \mathrm{t}-\phi)\} \\
& +2 \mathrm{~B} \cos \left(k \mathrm{kx}_{0}\right) \sum_{\mathrm{n}=1}^{\infty} \mathrm{J}_{2 \mathrm{n}}(\mathrm{kA}) \cos \{(2 \mathrm{n})(\omega \mathrm{t}-\phi)\}
\end{aligned}
$$

where $J_{n}(k A)$ is the Bessel function of the first kind and of order $n$. Omitting the steady component and rearranging give:

$$
\begin{aligned}
\mathrm{i}_{0}= & \mathrm{A}_{1}\{\cos (\phi) \sin (\omega \mathrm{t})-\sin (\phi) \cos (\omega \mathrm{t})\} \\
& +\mathrm{A}_{2}\{\sin (2 \phi) \sin (2 \omega \mathrm{t})+\cos (2 \phi) \cos (2 \omega \mathrm{t})\} \\
& +\mathrm{A}_{3}\{\cos (3 \phi) \sin (3 \omega \mathrm{t})-\sin (3 \phi) \cos (3 \omega \mathrm{t})\} \\
& +\mathrm{A}_{4}\{\sin (4 \phi) \sin (4 \omega \mathrm{t})+\cos (4 \phi) \cos (4 \omega \mathrm{t})\} \\
& +\ldots
\end{aligned}
$$

where the coefficients are defined by

$$
A_{n}= \begin{cases}-2 B \sin \left(k x_{0}\right) J_{n}(k A) & n=1,3,5,7 \ldots \\ 2 B \cos \left(k x_{0}\right) J_{n}(k A) & n=2,4,6,8 \ldots\end{cases}
$$

The coefficient, $A_{n}$, represents the amplitude of the $n$-th harmonic wave in the beat signal. Since $A_{n}$ may be positive or negative depending on the values of $\mathrm{kA}$ and $\mathrm{x}_{0}$, we may call it the signed amplitude.

Mathematically, Eq. (13) together with Eq. (14) is equivalent to the Fourier expansion of an ideal beat signal, in which the fundamental angular frequency is assumed to be the same as that of particle oscillation, $\omega$.

On the other hand, if actual beat signals are
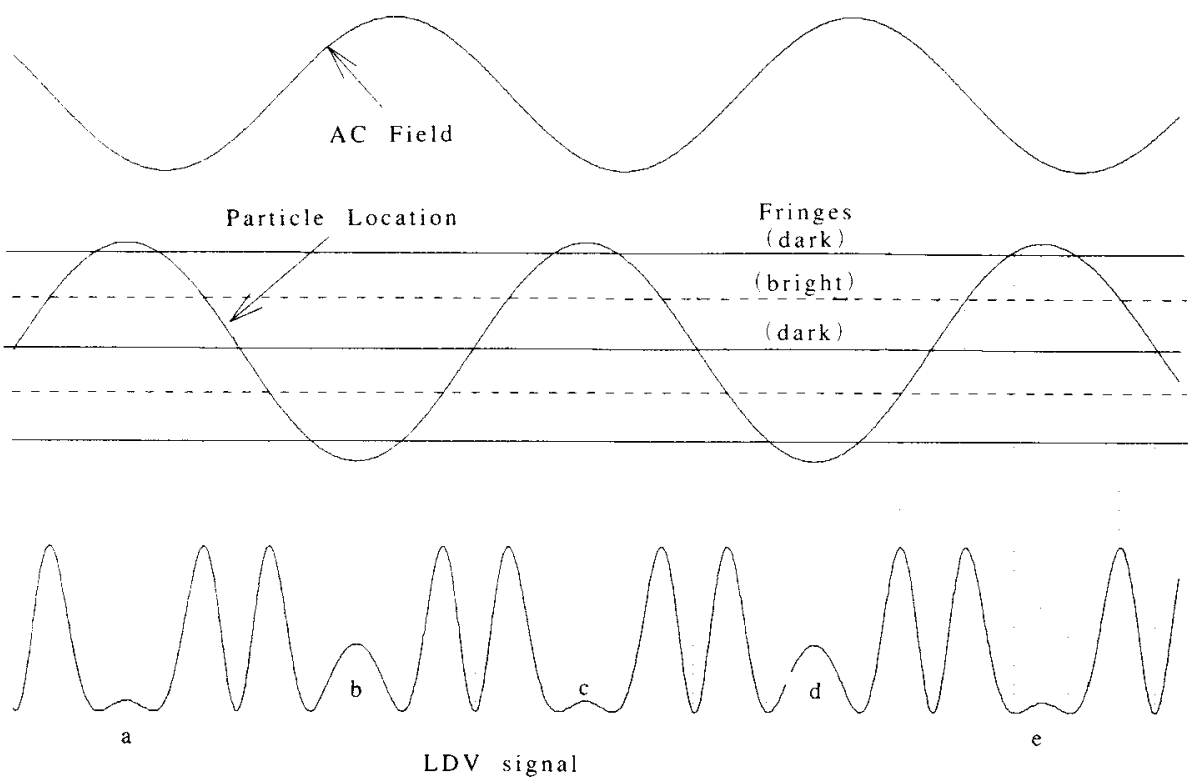

Fig. 3 Idel LDV beat signal 
numerically expanded into harmonic series of sine and cosine waves having a fundamental angular frequency $\omega$, the result may be written as follows:

$$
\mathrm{i}_{0}=\sum_{\mathrm{n}=1}^{\infty}\left\{\mathrm{F}_{\mathrm{sn}} \sin (\mathrm{n} \omega \mathrm{t})+\mathrm{F}_{\mathrm{cn}} \cos (\mathrm{n} \omega \mathrm{t})\right\}
$$

The coefficients in Eq. (15), $F_{\mathrm{sn}}$ and $\mathrm{F}_{\mathrm{cn}}$, can be calculated from the Fourier sine and cosine integrals of actual LDV signals, which may include a pedestal component. Taking the integration time $T$ as a multiple of the period of the particle oscillation, we can evaluate the following complex integral by some suitable numerical method explained later in $\S 4$.

$F_{n}=\left(\frac{2}{T}\right) \int_{0}^{T} i \exp (j n \omega t) d t, j=\sqrt{-1}, n=1,2,3 \ldots$

Then the real part and the imaginary part of $F_{n}$, respectively, give $F_{c n}$ and $F_{s n}$.

\subsection{Calculations for phase and amplitude measurements}

By comparing Eq. (13) with Eq. (15), we obtain the following relations for the coefficients :

$\mathrm{F}_{\mathrm{sn}}=A_{\mathrm{n}} \cos (\mathrm{n} \phi), \mathrm{F}_{\mathrm{cn}}=-\mathrm{A}_{\mathrm{n}} \sin (\mathrm{n} \phi) \mathrm{n}=1,3,5, \ldots$ $\left.\mathrm{F}_{\mathrm{sn}}=\mathrm{A}_{\mathrm{n}} \sin (\mathrm{n} \phi), \mathrm{F}_{\mathrm{cn}}=\mathrm{A}_{\mathrm{n}} \cos (\mathrm{n} \phi) \quad \mathrm{n}=2,4,6, \ldots\right\}$

Since $\phi$ is an angle between $90^{\circ}$ and $180^{\circ}$, both the above equations for $n=1$ and 2 can be used to obtain a unique value for $\phi$ i.e.,

$$
\left.\begin{array}{l}
\mathrm{n}=1 \quad \phi=\tan ^{-1}\left(\frac{-\mathrm{F}_{\mathrm{c} 1}}{\mathrm{~F}_{\mathrm{s} 1}}\right) 90^{\circ}<\phi<180^{\circ} \\
\mathrm{n}=2 \quad 2 \phi=\tan ^{-1}\left(\frac{\mathrm{F}_{\mathrm{s} 2}}{\mathrm{~F}_{\mathrm{c} 2}}\right) 180^{\circ}<2 \phi<360^{\circ}
\end{array}\right\}
$$

The method whereby $\phi$ can be obtained from two independent equations is quite advantageous because $A_{n}$ (and hence $F_{s 1}$ and $F_{c 2}$ in the denominators of the above equations) becomes zero when the trigometric functions and/or the Bessel functions are equal to zero (see Fig. 4) in Eq. (14). In practical calculation, it is recommended to use one of the equations for $n=1$ or $\mathrm{n}=2$ depending whether the absolute value of $F_{s 1}$ is greater than the absolute value of $F_{c 2}$ or not.

Equations (17) are then used to determine $A_{n}$ 's with their appropriate signs from $F_{\mathrm{sn}}$ 's and $F_{\mathrm{cn}}$ 's for several values of n. Further, from Eq. (14), the ratio of $A_{n+2}$ to $A_{n}$ is obtained as follows:

$$
\frac{A_{n+2}}{A_{n}}=\frac{J_{n+2}(k A)}{J_{n}(k A)}=Q_{n}(k A)
$$

This is a function of only $\mathrm{kA}$, and the particle amplitude A can be obtained from this ratio. Since the Bessel functions are damped oscillating functions as shown in Fig. 4, however, the functions, $Q_{n}$ 's, defined by their ratios are not monotonous. As a result, the inverse functions, $\mathrm{Q}_{\mathrm{n}}{ }^{-1}$ 's, become multi-valued functions with innumerable branches. In Fig. 5, the first and second branches of $Q_{n}$ 's for several values of $n$ are shown. Therefore, we will have an infinite number of values for $\mathrm{kA}$ even for a given single value of $Q_{n}$.

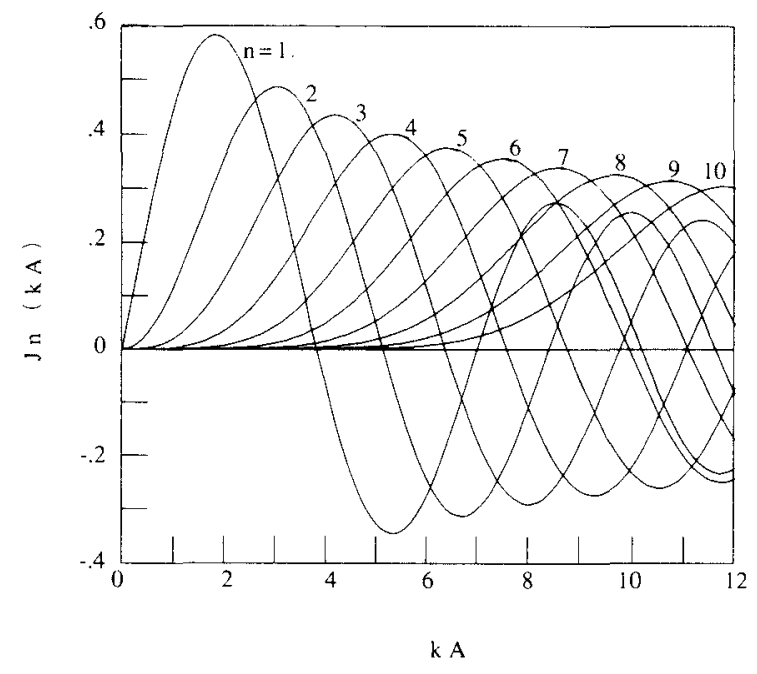

Fig. 4 Bessel function of the first kind

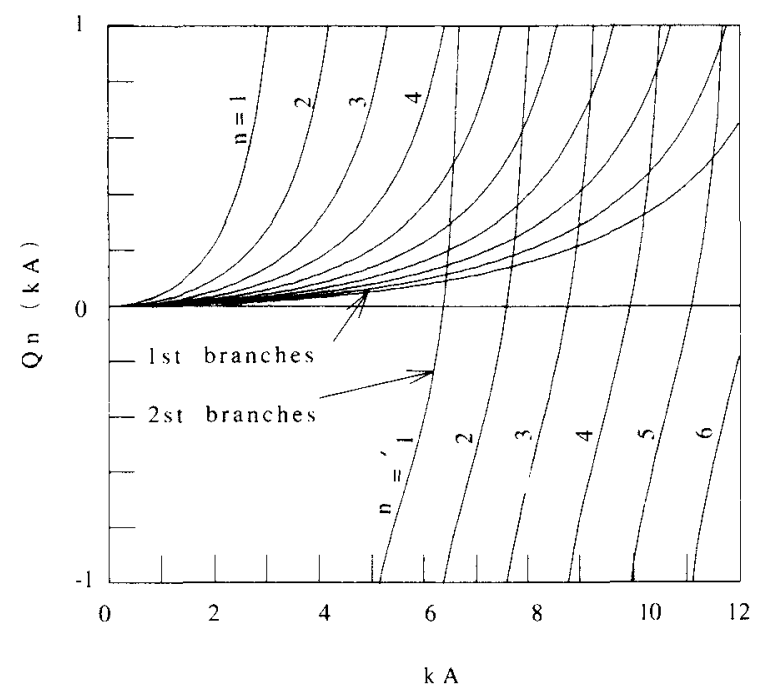

Fig. 5 First two branches of the Function $Q_{n}(k A)$ 
As can readily be seen from Fig. 5, the inverse function, $Q_{n}{ }^{-1}$, gives a single value if the value of the inverse function is confined to the range between the first zero (i.e., $\mathrm{kA}=0$ ) and the second zero of $Q_{n}$. We may call this range "the principal range" of $\mathrm{Q}_{\mathrm{n}}{ }^{-1}$. Since the upper limit of the principal range becomes large for a large $\mathrm{n}$, any large value of $\mathrm{kA}$ can be included in the principal range for $\mathrm{n}$ greater than a certain integer. Therefore, if we can select a suitable integer for $\mathrm{n}$ from the beat signal analysis, the proper value of $\mathrm{kA}$ can be uniquely found in the principal range for $\mathrm{Q}_{n}{ }^{-1}$. From the analysis of Eqs. (13) and (14) together with Figs. 4 and 5, the most suitable integer for $n$ turns out to be of the order of the strongest harmonic wave in the beat signal.

This provides a simple method for determining $\mathrm{kA}$ : Find the strongest amplitude, $A_{n}$, among the signed amplitudes calculated using Eq. (16). Obtain the principal value (i.e., minimum value in the present calculation) for $\mathrm{kA}$, which satisfies $\frac{A_{n+2}}{A_{n}}$, n being of the order of the strongest harmonic wave. The value of $\mathrm{kA}$ thus obtained gives the correct amplitude of the particle vibration.

\section{Wave analysis of actual beat signal}

The above theoretical results have been deduced with the assumption that the LDV signals are emitted from ideal fringes. Beat signals from actual LDV fringes are distorted by many sources, e.g., pedestal term, speckles, intensity fluctuations in laser beam, illuminated foreign particles, air flow disturbance in LDV fringes, and so on.

The authors have kept a good deal of LDV signal data digitally recorded in floppy disks obtained from the previous experiment ${ }^{3)}$. The experiment was carried out with DOP droplets of $1.29 \mu \mathrm{m}$ in radius, which were produced by an aerosol generator of the vibrating orifice type. The particle vibration frequency was approximately $6 \mathrm{kHz}$ and therefore the phase lag was $127^{\circ}$. The LDV and the AC field signals were sampled by a two-channel, 8-bit digitizer for $1 \mathrm{~ms}$ duration at a sampling frequency of 1 $\mathrm{MHz}$, and were recorded as a series of 2000 words binary data. An example of a reproduced $\mathrm{AC}$ field and LDV signals, respectively, is shown in the top and middle curves in Fig. 6.
Reverse points (corresponding to points a, b, c, ... in Fig. 3) may be found in the LDV signal, although they are not very clearly noticeable. The particle amplitude for this LDV signal has been regarded as being at the sensible lower limit of the reverse points for the computer algorithm used in the previous method mentioned in $\S 2.3$.

As stated in Eq. (16), the integration time, T, should be taken as a multiple of the period of the particle vibration, so that the LDV signal can be decomposed into a harmonic series of the fundamental frequency, i.e., the frequency of the particle vibration. This requirement is quite understandable for ideal LDV signals in which the fundamental frequency is really reflected in their waveforms as shown in Fig. 3. On the contrary, actual LDV signals may be so distorted that the fundamental wave cannot be recognized in LDV signals; in fact it is very hard to distinguish the fundamental wave in the actual LDV signal shown in Fig. 6.

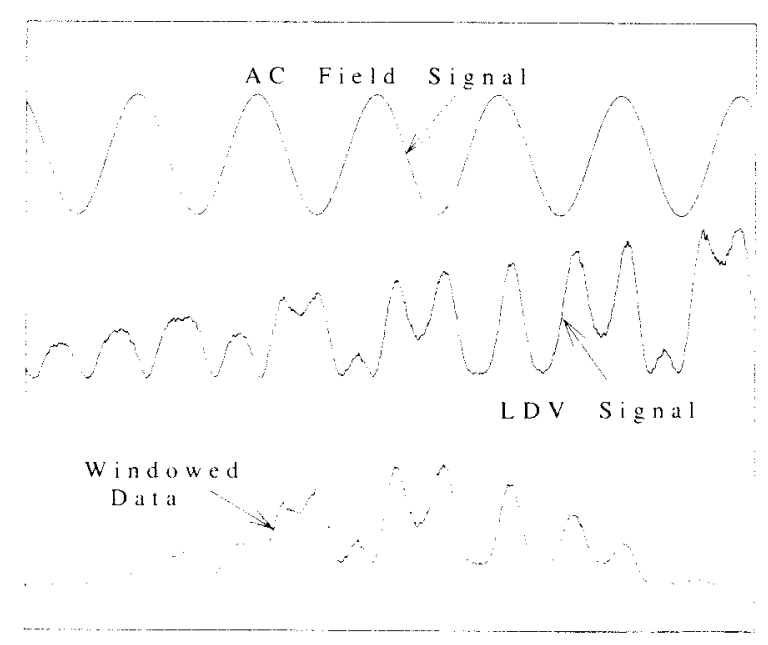

Fig. 6 Example of observed LDV signal

For the reason mentioned above, it is not certain that precise information for obtaining the phase and amplitude of a particle vibration can be extracted from actual LDV signals with the aid of Eq. (16), in which the fundamental wave plays an essential role. Nevertheless, the authors intend to analyze actual LDV signals on the basis of the mathematical discussions given in the preceding sections.

Here the mathematical role of the angular frequency, $\mathrm{n} \omega$, in Eq. (16) is reconsidered so as to obtain a virtually continuous spectrum of the 
LDV signal. Let's suppose a fictitious signal with long dummy data of zero (or constant) intensity attached before and after the actual recorded data of an LDV signal. The integration time $T$ for this fictitious signal becomes very long for $\omega=\left(2 \frac{\pi}{\mathrm{T}}\right)$ to approach zero, and nw can actually be regarded as a continuous variable. Since relative values of $F_{n}$ for various $\mathrm{n} \omega$ are in question in the present calculation, the proportionality $\frac{2}{\mathrm{~T}}$ in Eq. (16) is not an essential factor. In this sense, the integration may be performed for an arbitrary angular frequency, $\Omega$, regardless of the actual signal duration, $T$, by simply substituting $\Omega$ for $\mathrm{n} \omega$ in Eq. (16), provided that $\Omega$ is fairly larger than $\frac{2 \pi}{\mathrm{T}}$.

The amplitude spectrum of the LDV signal shown in Fig. 6 was calculated in this manner. If the LDV signal had been ideal, we would have had line peaks at several multiples of 6 $\mathrm{kHz}$, the driving frequency of the particle oscillation. In contrast to the ideal case, the actual spectrum shown in Fig. 7 displays an appreciable broadening and has dips at multiple frequencies of $6 \mathrm{kHz}$. The broadening is a result of a rather too short duration ( $1 \mathrm{~ms}$ ) of the recorded LDV signal. This can be understood in terms of the boxcar window function discussed in the theory for spectrum analysis? ${ }^{7}$.

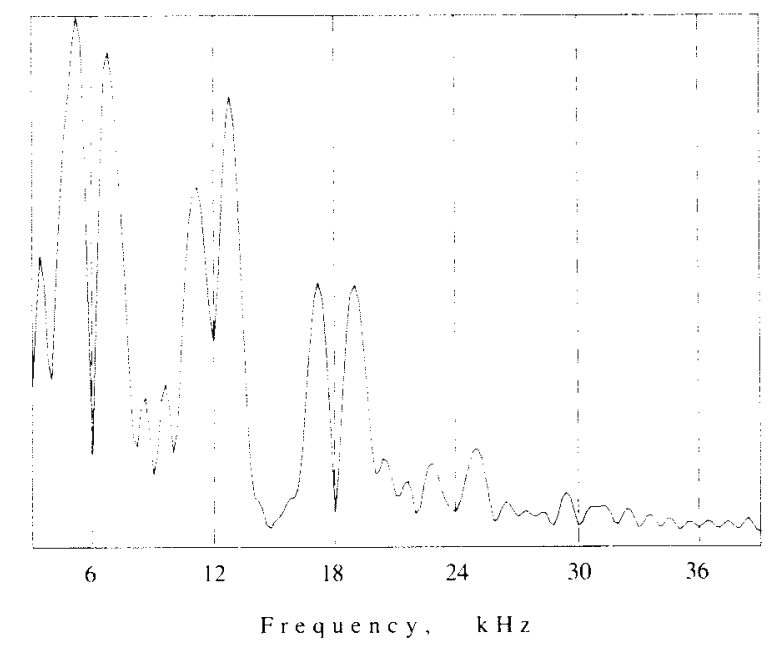

Fig. 7 Amplitude spectrum of the LDV signal in Fig 6

The dips can be explained by the effect of the particle drift velocity perpendicular to the fringe planes as follows : If the particle vibration in the fringes accompanies a steady drift velocity, the beat frequency becomes higher in the half period of the vibration where the particle velocity is enhanced by the drift velocity, and then becomes lower in the second half period. Such a frequency shift in the beat signal results in a separation of every peak in the spectrum of an actual LDV signal into pairs of peaks. The peak separation can be shown mathematically by simply replacing $\mathrm{kx}_{0}$ with $\mathrm{k}\left(\mathrm{x}_{0}+\mathrm{Ut}\right)$ in $\mathrm{Eq}$. (12). In the spectrum of an actual LDV signal with broadening, peak separation would display a dip at the middle frequency of every pair of peaks.

Judging from the above reasoning, Fig. 7 may be acceptable as a reasonable LDV spectrum. Hence $F_{\mathrm{sn}}$ and $\mathrm{F}_{\mathrm{cn}}$ for $6 \mathrm{kHz}$ and $12 \mathrm{kHz}$ were calculated using Eq. (16) to obtain $\phi$ from Eq. (18). However, the result $\left(=121.7^{\circ}\right)$ differed considerably from the expected value, $127^{\circ}$. Such calculations for 60 sets of data collected under the same experimental condition gave a rather unsatisfactory result. This is assumed to be caused by data truncation; as shown in Fig. 6, at the beginning and end of the recorded signal, there are sudden changes in signal intensity which may give rise to disturbances in the calculated spectrum.

In waveform data processing techniques, time series data are often multiplied by a function of time called window function before spectral analysis, so that the resultant data fade in and out smoothly at the beginning and ending portions of the truncated signal. It is well known that disturbances in a spectrum can be smoothed out using such a window function, which averages the spectrum intensity over a certain frequency width. In the present calculation, a Hanning window ${ }^{7)}$ is adopted, which takes the form $\frac{\left\{1-\cos \left(\frac{2 \pi t}{\mathrm{~T}}\right)\right\}}{2}$ where $\mathrm{T}$ is the signal duration and $t$ is the time of the sampling point. $(\mathrm{t}=0 \sim \mathrm{T})$. Thus the original data shown in the middle curve in Fig. 6 was transformed into the windowed data shown in the bottom curve.

The spectrum of the windowed data is shown in Fig. 8. We can see that many phantom peaks observed in Fig. 7 completely disappeared. As a result of this improvement, the phase lag calculated from the windowed data gave a value very close to the expected value, $127^{\circ}$. Then phase lags for the 60 sets of recorded data were 
recalculated using this window technique. The phase distribution thus obtained was converted into the radius distribution of the monodisperse DOP droplets obtained from the aerosol generator. The average and the standard deviation of the radius distribution, respectively, were 1.289 $\mu \mathrm{m}$ and $0.0188 \mu \mathrm{m}$. These values coincided very well with the expected values for the operational conditions of the aerosol generator. In this way, the efficacy of the Hanning window is really advantageous for the phase measurement, although this fact doesn't necessarily mean that the spectrum calculated using the window function gives the true spectrum of the signal.

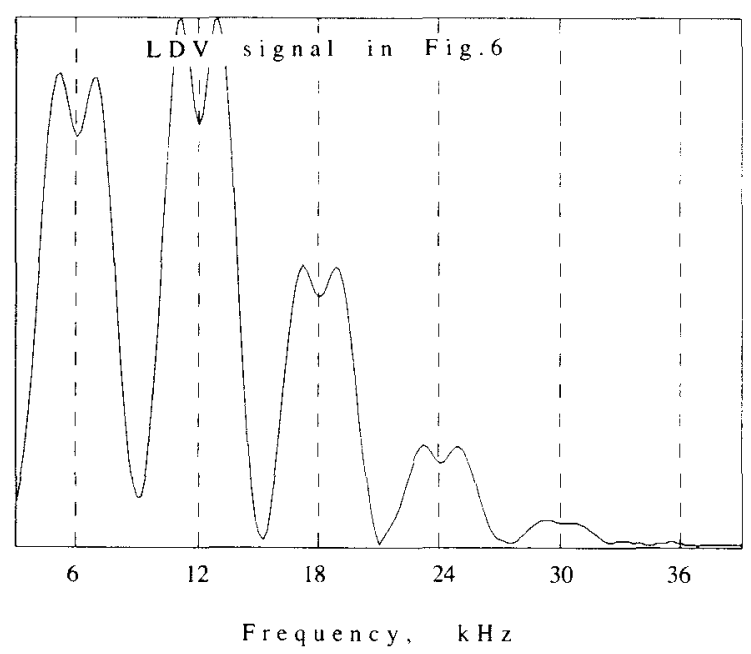

Fig. 8 Effect of Hanning window on the spectrum

It should be noted here that the frequency of the AC field, on which the phase calculation is based, must be measured precisely; only a few percent error in the frequency results in an unacceptable error in the phase measurement. And, another point for calculating the phase lag, one of the equations in Eq. (18) should be properly selected with due care as mentioned in the related section.

To obtain the amplitude of a particle vibra. tion, the signed amplitudes of the harmonic waves, $A_{n}$ 's, were calculated using Eqs. (16) and (17) for $\mathrm{n}$ from 1 to 12. This range of $\mathrm{n}$ is suitable when $\frac{A}{\delta}$ is less than 2 . For the measurement of a larger amplitude, the calculations must be extended to higher order harmonics. However, the present method loses its advantage over the previous method when the ampli- tude becomes greater than the fringe spacing. Therefore, in comparison with normal spectrum analyses, a very limited number of frequencies for the analysis will suffice for the present method.

The amplitude of a particle vibration depends on the particle charge. DOP droplets obtained from the aerosol generator used in the experiment were generated by breaking up periodically a liquid jet spouted from a vibrating platinum micro orifice. An experiment on electrostatic charges of particles obtained from the aerosol generator showed that the DOP droplets had a constant positive charge by spontaneous triboelectrification ${ }^{8}$. Therefore, it is not surprising that the particle amplitude calculated from the experimental data for the same operational conditions of the generator shows almost a constant amplitude; in fact, the average of $\frac{\mathrm{A}}{\delta}$ (where $\delta=8.68 \mu \mathrm{m}$ ) was found to be 0.431 with a standard deviation of 0.020 for the same data as those used in the phase measurement. Although we have no means to confirm the correctness of the above result, 47 sets of data out of 60 could be analyzed by the previous method $^{3)}$ and a reasonable agreement with the above result was observed; the result obtained by the previous method was $\frac{\mathrm{A}}{\delta}=0.426$ with a standard deviation of 0.012 .

As stated before, the drift velocity of a particle gives rise to peak separation and yields dips in its LDV spectrum at the harmonic frequencies of the particle vibration. Since the present method totally relies on $\mathrm{F}_{\mathrm{n}}$ 's in Eq. (16) calculated for the harmonic frequencies, the depths of the dips greatly affect that accuracy of the measurement. We see from Figs. 7 and 8 that the dips can be much flattened when a Hanning window is used. However, the efficacy of the window is of course limited to the case where the drift velocity is not too large. In the previous method, the drift velocity could be calculated from the LDV signal to compensate for its effect. Further, when the compensation was discriminated to be rather unsuccessful, the data was automatically abandoned. This is the major reason why only 47 data out of 60 could be analyzed, and as a result, the standard deviation obtained by the previous method was slightly smaller. 
The peak separation shown in Figs. 7 and 8 corresponds to a drift velocity of $0.78 \mathrm{~cm} / \mathrm{s}$. The maximum drift velocity in the experiment was found to be $1.5 \mathrm{~cm} / \mathrm{s}$. (The velocity is of course the velocity component perpendicular to the fringe planes.) Since the fringe planes are formed parallel to the plate electrodes, it may not be difficult to keep the drift velocity lower than this level.

\section{Numerical integration}

Eq. (16) is no other than the formula of the Fourier expansion for periodic functions. The only difference in the sign of the exponential term is not a matter of significance for the present discussion. In contrast to conventional LDV systems, a digital computer is used for the waveform data processing. As a consequence, the LDV signal is converted into a time series of digital data for the numerical processing, which naturally resembles very much the DFT (Discrete Fourier Transformation). Therefore techniques for the DFT such as the FFT algorithm may be applicable. In this section, however, it is pointed out that the calculated result using the DFT is not directly applicable to the present method.

In the DFT (and hence in the FFT) algorithm, the whole integrand in Eq. (16), i exp (jnct), is approximated to a linear function in an interval between neighboring data points so as to obtain an approximate value of the integral for the single interval. When nw is so small that exp (jnwt) remains almost constant in each interval, the sum of representative values for all intervals may give a value close to the integral of Eq. (16). However, for a large value of no, the DFT algorithm may not be a reasonable approximation of the integral. Nevertheless the resultant DFT coefficients can exactly reproduce the original data, $\mathrm{i}$, using the inverse DFT. Here we must remember the fact that the reproduction of original data using the inverse DFT is possible only for the moments which exactly correspond to the sampling points. If we calculate the data, i, for any moment between the sampling points, the result may give a quite unreasonable value for $i$. It follows that the DFT may not be regarded as a reasonable approximation of the Fourier integrals of the functions which vary with time smoothly and almost linearly between the sampling points.
To obtain acceptable values for $F_{n}$ numerically from Eq. (16), only the LDV signal, i, and not $i \exp (j n \omega t)$, should be approximated to a linear function between neighboring data points; $i=a t+b$. The resultant integrand, (at + b) $\exp (j n \omega t)$, is integrated algebraically over a single data interval, and then the results are summed up to obtain the total integral of Eq. (16). The calculation is rather tedious but the final result can be summarized in the following simple conclusion :

The coefficient, $F_{n}$, calculated for $\Omega$ from the above approximation can be obtained by multiplying the coefficient of the DFT by a factor of $2\{1-\cos (\Omega \Delta t)\}$ $(\Omega \Delta t)^{2}$

interval. The coefficients thus calculated coincide well with the Fourier coefficients in Eq.

(15) for $\Omega(=\mathrm{n} \omega)$ lower than $\frac{\pi}{\Delta \mathrm{t}}$.

List 1 Example program of Fourier integrals for extracting the fundamental and harmonic waves from LDV signal.

* * Fourier Sin/Cos Integrals ** cosine $=\operatorname{REAL}(\mathrm{Zn})$, sine $=\operatorname{IMAG}(\mathrm{Zn})$

note : $\mathrm{pi}=\pi, \mathrm{j}=\sqrt{(-1)}$

$\operatorname{CEXP}(\mathrm{j} * \theta)=\cos (\theta)+\mathrm{j} * \sin (\theta)$

$\mathrm{HanW}(\mathrm{K}):$ Windowed data(real)

$\mathrm{Han} W(0)=\mathrm{Han} W($ Kdata +1$)=0$

FFreq : Fundamental Frequency

St : Sampling time interval

$\mathrm{j}=\operatorname{CMPLX}(0.0,1.0)$

$\mathrm{Dth}=2.0 *$ pi $*$ FFreq $*$ St

Do $200 \mathrm{Nf}=1$, NFourier

ZDthNf $=\mathrm{CEXP}(\mathrm{Dth} * \mathrm{Nf} * \mathrm{j})$

$\mathrm{ZW}=\mathrm{ZDthNf}$

$Z$ sum $=0.0$

300

Do $300 \mathrm{~K}=1$, Kdata

$Z$ sum $=\operatorname{HanW}(\mathrm{K}) * Z \mathrm{~W}+Z$ sum

$Z \mathrm{~W}=\mathrm{ZW} * \mathrm{ZDthNf}$

(Zsum : DFT)

linear interpolation between data points. $200 \mathrm{Zn}(\mathrm{Nf})=(1.0-\operatorname{REAL}(\mathrm{ZDthNf})) /(\mathrm{Nf} * \mathrm{Nf}) * \mathrm{Zsum}$

List 1 is an example of a Fortran program used for the calculation, in which non-essential constants are omitted. In this program, the variables starting with $Z$ together with $\mathrm{j}$ are complex variables. The loop enclosed by the statement number 300 is no other than the DFT. 
The phase rotator is $Z \mathrm{~W}$. The complex variable, Zsum, is for the result calculated by DFT, and is multiplied at the statement 200 by a factor, $\frac{(1.0-\mathrm{REAL}(\mathrm{ZDthNf}))}{(\mathrm{Nf} \cdot \mathrm{Nf})}$, which corresponds to the factor, $\frac{\{1-\cos (\Omega \Delta t)\}}{(\Omega \Delta t)^{2}}$. The FFT algorithm is not used in this program because only a limited number of frequencies are required to be analyzed.

\section{Simulated performance}

\subsection{Beat signal generation}

In this simulation model, the same optical system as described in the previous paper ${ }^{3)}$ was considered; $\mathrm{b}_{0}, \alpha, \delta$, and $\lambda$ in Eqs. (9) and (10), respectively, were $200 \mu \mathrm{m}, 0.0725 \mathrm{rad}\left(4.18^{\circ}\right)$, $8.68 \mu \mathrm{m}$, and $0.6328 \mu \mathrm{m}$. A particle to be traced in the simulation was initially located at a position $\left(\mathrm{x}_{0}, \mathrm{y}_{0}, z_{0}\right)$ in Fig. 2; $\mathrm{x}$ and $z_{0}$, respectively, were randomly positioned within $\pm 1.5 \mathrm{~b}_{0}$ and $\pm 1.5 \frac{\mathrm{b}_{0}}{\sin \left(\frac{\alpha}{2}\right)}$, while $\mathrm{y}_{0}$ was a constant,

i.e., $-1.5 b_{0}$. This area for initial positions was wide enough to cover the whole measurement control volume of the simulated LDV system. From an initial position thus chosen randomly, a particle to be traced was transported in the $y$ direction by a steady air flow with a velocity of $30 \mathrm{~cm} / \mathrm{s}$. The frequency of the particle vibration in the $\mathrm{x}$ direction was fixed at a constant value of $12 \mathrm{kHz}$; the phase lag was randomly given as an angle between $100^{\circ}$ and $170^{\circ}$. The amplitude was set at a random value within a range between $0.05 \delta$ and $1.2 \delta ; \delta$ is the fringe spacing. The drift velocity in the $\mathrm{x}$ direction, which causes peak separation in the spectrum, was given randomly within $\pm 2 \mathrm{~cm} / \mathrm{s}$.

Under the above condition, beat signals for the simulation were calculated using Eq.(9) for the trajectories of about 42,000 particles. In the simulated system, an 8-bit digitizer was assumed to be used for recording the beat signal. Then the proportionality in Eq.(9) was assumed to be 128 so that the signals could be expressed in 8 -bit integer data by omitting figures below the decimal point.

\subsection{Trigger rate and count efficiency}

The digitizer was triggered when the intensity of the signal exceeded a certain level, which was assumed to be $10 \%$ of the maximum intensity. The ratio of the number of particle trajectories which triggered the digitizer to the total number of particles traced is tentatively named 'trigger rate'.

As the digitizer was triggered whenever the signal intensity exceeded the threshold, some of the triggered signals had too poor visibility for the waveform data processing. For instance, when a particle passed across a single laser beam near its center axis but outside the fringe area, the signal was intense enough to trigger the digitizer but wholly void for the processing. Since the illuminating light intensity distribution in the beam cross section was assumed to be Gaussian (as usual in actual LDV optical systems), such a void signal was modulated by the frequency of the particle oscillation. This situation is true also in actual LDV systems.

It follows that a high-pass filter with a cut off frequency higher than the frequency of the particle oscillation is necessary for the removal of such void signals. However, the use of a high pass filter would totally spoil the present method because one of the most important frequency components in normal signals comprises the frequency of the particle oscillation. Consequently, the computer program used for the waveform data processing should provide a function for the discrimination between acceptable signals and void signals for the processing. For this reason, not all of the triggered signals are supposed to be processed. The ratio of the signals adopted for the processing to the total triggered signals is tentatively termed 'count efficiency'.

The trigger rate and the count efficiency for the previous method depend appreciably on the particle amplitude when the amplitude is smaller than the fringe spacing. The trigger rate is of course affected by the area selected for the initial position of the particles. Therefore, only the relative change in the trigger rate in response to the amplitude is of significance. Referring to the changes in the trigger rate and the count efficiency, the measured amplitude distribution (and hence the charge distribution) can be corrected. However the particle size distribution must not be calculated from the measured phase lag distribution unless the breadth of the distribution is very narrow. This is because the actual signal intensity depends 
highly on the particle size ; this fact means that the trigger rate and the count efficiency depend also on the particle size.

\subsection{Sampling frequency and number of sam- ple points}

As stated above, the frequency of the particle vibration in this simulation was $12 \mathrm{kHz}$ and its maximum amplitude was assumed to be $1.2 \delta$. These gave an estimation of the maximum frequency to be analyzed as follows:

The maximum $\mathrm{kA}$ is $2 \pi \times 1.2=7.5$, and the absolute value of the signed amplitude $A_{n}$ in $\mathrm{Eq}$. (14) takes its largest value at $\mathrm{n}=6$ or 7 for $\mathrm{kA}=$ 7.5. Since we need $A_{n+2}$ to obtain the particle amplitude, the possible maximum of the harmonic frequency to be analyzed is $(n+2) \times$ $12 \mathrm{kHz}=108 \mathrm{kHz}$ for $\mathrm{n}=7$.

The sampling frequency of the digitizer must be higher than twice this maximum frequency, i.e., $216 \mathrm{kHz}$. This is the minimum value for the sampling frequency required by the sampling theorem, therefore a $500 \mathrm{kHz}$ sampling $(\Delta \mathrm{t}=2$ $\mu \mathrm{s})$ was selected for the simulation to avoid aliasing. ${ }^{9)}$

The signal time duration for the analysis, $T$, is proportional to the number of the sample points. Naturally, we may expect a better accuracy of the measurement with a larger number of sample points, N. Since T affects the window characteristics, however, an additional problem arises if a too large number of sample points is carelessly used without reducing the drift velocity of a lower level.

The window function averages the spectrum over a certain frequency range, which is inversely proportional to $\mathrm{T}$. Therefore, a larger $\mathrm{T}$ or $\mathrm{N}$ yields a higher spectrum resolution. For the present method of analysis, however, a very high resolution is not necessarily favorable. This is because a high resolution exaggerates the dips in the spectrum caused by the drift velocity at the frequencies required for the analysis.

In the previous experiment, the signal time duration was $1 \mathrm{~ms}$ for a maximum drift velocity of $1.5 \mathrm{~cm} / \mathrm{s}$. Although these values might not be standard, $\mathrm{T}$ was tentatively chosen to be $1 \mathrm{~ms}$ (and hence $\mathrm{N}=512$ ) for this simulation for a maximum drift velocity of $2 \mathrm{~cm} / \mathrm{s}$.

\subsection{Discrimination of beat signal}

As explained in $\S 5.2$, not all the triggered signals were suitable for data processing. In this simulation, beat signals were discriminated in the following manner according to a measure for visibility of the beat signals.

The visibility defined by Roberds ${ }^{5)}$ was $\frac{\mathrm{V}_{0}}{\cosh \left(\frac{2 \times z \alpha}{\mathrm{b}_{0}{ }^{2}}\right)}$; this definition can be understood from Eq.(9). However, it is not easy to evaluate the visibility thus defined from actual LDV signals. In this simulation,

$\mathrm{V}=\frac{\sum_{n=1}^{9}\left|F_{n}\right|}{\left|F_{0}\right|}$ was adopted for the visibility, where $\left|F_{n}\right|$ 's are absolute values of the complex quantities calculated using Eq.(16) for $n$ from 0 to 9 . This definition of visibility may be considered as being the ratio of the sum of the intensities of the significant components to the total intensity of the signal.

The adoption of a small value for the visibility threshold increases the possibility of miscalculation, and decreases the accuracy and reliability of the measuring system. But the visibility becomes lower and lower as the particle amplitude decreases. When $\frac{\mathrm{A}}{\delta}=0.1, \mathrm{~V}$ is less than 0.3 for the optimal condition; the visibility becomes optimal when the particle vibrates at the center of the fringes. Therefore, too large a value for the threshold decreases unacceptably the count efficiency for small amplitudes. The threshold was assumed to be 0.1 for the simulation.

Although this value might be too large for the measurement of small amplitudes, miscalculations still occurred with this value. When a particle passed through some portion of the fringes having a very weak contrast, the fundamental frequency component became highest when the particle amplitude was large enough to make some harmonics stronger than the fundamental if the particle had been in normal fringes ; this was an effect of the Gaussian distribution in beam intensity stated previously and led to miscalculation of the particle amplitude. To avoid this sort of miscalculation, the fundamental component was ignored if harmonics higher than the 4 th had appreciable intensities. 


\subsection{Simulated results}

About 42,000 particle trajectories were traced to examine the effect of their amplitude on the trigger rate and on the count efficiency. The result is shown in Fig. 9. The trigger rate depends little on the amplitude. In contrast to this, the count efficiency decreases considerably with a decrease in amplitude. This is an inevitable result of the discrimination of signals. However, the change occurs fairly smoothly down to $\frac{A}{\delta}=0.1$, and compensation for obtaining the amplitude distribution may be possible. This is an advantage over the previous method.

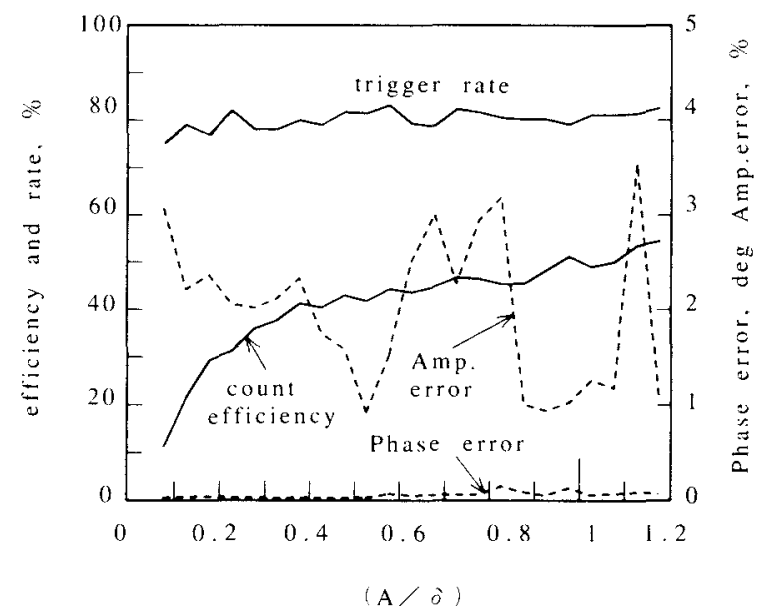

Fig. 9 Simulated results

In Fig. 9, the standard deviations of the error in the phase and of the relative error in the amplitude are also shown. The amplitude error varied widely with the amplitude; the error might have resulted from miscalculation caused by incomplete discrimination. Nevertheless, the error may well be considered to be within an acceptable level for normal purposes. On the other hand, the phase error was very small and was of the order of $0.05^{\circ}$ on average. It follows that a fairly accurate particle size can be calculated from a rather small phase lag, caused by a rather too low frequency for the particle size. Or in other words, we may use a considerably low frequency even for small particles.

As explained before, the drift velocity of a particle in the $\mathrm{x}$ direction leads to peak separation and causes dips at the analyzed frequencies. As a result, LDV signals observed for large drift velocities tended to be classified as having too poor a visibility. Fig. 10 shows the effect of the drift velocity on the count efficiency, i.e., the area below the curve over $\mathrm{U}=\mathrm{U}$ $\sim \mathrm{U}+\mathrm{dU}$ gives the number of particles being processed of which the drift velocity was in the range between $U$ and $U+d U$. Since the drift velocity was distributed uniformly in this simulation, it is observed from the figure that the count efficiency suddenly decreases for $U$ greater than $1.6 \mathrm{~cm} / \mathrm{s}$.

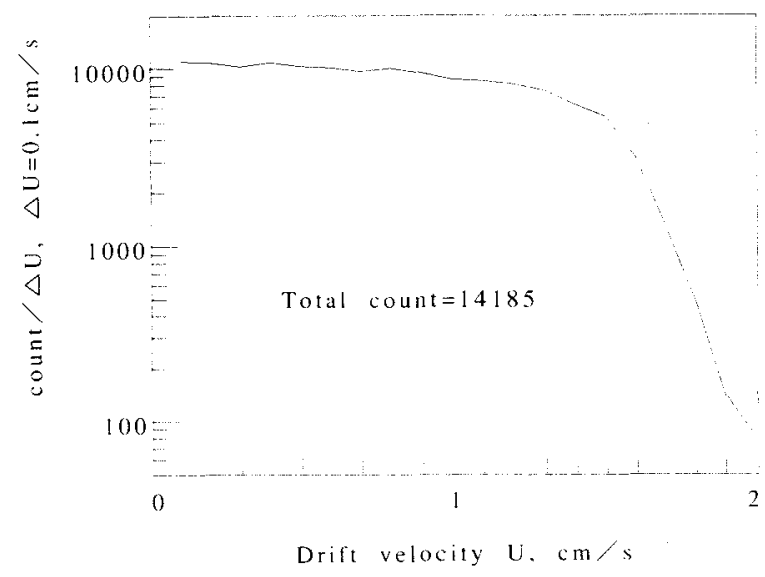

Fig. 10 Effect of drift velocity on count efficiency

The simulated results for U less than $1 \mathrm{~cm} / \mathrm{s}$ were examined to determine whether the accuracy of the measurement could be improved for smaller drift velocities. However, no appreciable differences could be found in the amplitude and phase errors. Therefore we may conclude that the drift velocity affects mainly the count efficiency and slightly the accuracy of the measurement.

As stated in $\S 5.3$, we can expect a better accuracy with longer signal time durations, which lead to a higher spectrum resolution as a by-effect. To maintain the count efficiency at a reasonable level for a high resolution of the spectrum, we must decrease the drift velocity. Keeping these points in mind, we carried out another simulation test for $\mathrm{T}=2 \mathrm{~ms}$ or 1024 sample points ( i.e., twice the time duration of the first simulation). The maximum drift velocity was assumed to be $1 \mathrm{~cm} / \mathrm{s}$ (i.e., half the maximum of the first simulation) and $b_{0}, 400$ $\mu \mathrm{m}$ (i.e., twice the radius of the first simulation). Other simulation parameters remained unchanged. The result showed that the effect of the amplitude on the trigger rate and on the count efficiency was almost the same as that shown in Fig. 9. However the errors in both the amplitude and phase measurements decreased to less than half the errors observed in the first 
Wsimulation. The effect on the count efficiency was quite similar to that shown in Fig. 10 except that the drift velocity should be read as being half that indicated in Fig. 10.

\section{Conclusions}

The Fourier analysis is applied to waveform data processing of the LDV signal emitted by an oscillating particle. The amplitude and the phase lag of the particle oscillation can be calculated using a spectrum analysis of the LDV signal. If a charged particle is forced to oscillate under the effect of an AC electric field, the size and the charge of the particle can be determined from the phase lag and the amplitude.

The accuracy of the phase lag measurement is believed to be so high that a frequency of the order of $10 \mathrm{kHz}$ may be used for the measurement of a particle size as small as $0.3 \mu \mathrm{m}$. As for the amplitude measurement, the proposed method provides a convenient means for the determination of rather small amplitudes of around $1 \mu \mathrm{m}$. Both features mentioned above may be helpful for the measurement of small electrostatic charges on particles finer than 1 $\mu \mathrm{m}$.

A longer signal duration can improve the accuracy of the measurement. The sensibility of the amplitude may be increased by decreasing the LDV fringe spacing. However, both modifications may lead to a drop in the count efficiency to a great extent because peak separation by drift velocity is exaggerated for longer signal durations and/or smaller fringe spacings. Consequently, the drift velocity caused by thermal convection and air flow turbulence should be eliminated as carefully as possible.

The polarity of a particle charge cannot be determined by the present method. Recently, we came upon a possible method to avoid this shortcoming by superposing on purpose a constant drift velocity on the vibrating motion of the particle.

\section{Nomenclature}

A : amplitude of particle oscillation, $m$

$\mathrm{A}_{\mathrm{n}} \quad$ : signed amplitude of $\mathrm{n}$-th harmonic, Ampere

$a_{n} \quad$ : constants in Eq.(6), dimensionless

B : proportionality, Ampere

$\mathrm{b}_{0} \quad$ : radius of laser beam $\left(\frac{1}{\mathrm{e}^{2}}\right.$ point, $\mathrm{m}$

C : Cunningham correction factor, dimensionless

$D_{\mathrm{p}} \quad$ : particle diameter, $\mathrm{m}$

$\mathrm{E} \quad$ : amplitude of AC field, $\frac{\mathrm{V}}{\mathrm{m}}$

$F_{n} \quad$ : complex quantity for expressing Fourier sine and cosine integrals defined by Eq.(16), Ampere

$\mathrm{F}_{\mathrm{cn}} \quad$ : real part (cosine component) of $\mathrm{F}_{\mathrm{n}}$, Ampere

$\mathrm{F}_{\mathrm{sn}} \quad$ : imaginary part (sine component) of $F_{n}$, Ampere

i : output current, Ampere

$i_{b} \quad$ : beat signal current, Ampere

$i_{\circ} \quad$ : oscillating component in output sig. nal, Ampere

$\mathrm{J}_{\mathrm{n}} \quad$ : Bessel function of the first kind and of order $\mathrm{n}$

$\mathrm{j}=\sqrt{-1}$

$\mathrm{k}=\frac{2 \pi}{\delta}$, fringe wave number, $\frac{1}{\mathrm{~m}}$

$\mathrm{N} \quad$ : number of data points

$\mathrm{n} \quad$ : order of harmonics

$\mathrm{Q}_{\mathrm{n}}(\mathrm{kA})=\frac{\mathrm{J}_{\mathrm{n}+2}(\mathrm{kA})}{\mathrm{J}_{\mathrm{n}}(\mathrm{kA})}$

q : particle charge, Coulomb

$\mathrm{T}$ : signal time duration or integration time, $\mathrm{s}$

$\Delta \mathrm{T} \quad$ : sampling time interval of digitizer, $\mathrm{s}$

t : time, $\mathrm{s}$

$\mathrm{U}$ : drift velocity of particle in $\mathrm{x}$ direction, $\frac{\mathrm{m}}{\mathrm{s}}$

$\mathrm{V} \quad$ : visibility, dimensionless

$\mathrm{V}_{0} \quad$ : visibility parameter, dimensionless

$\mathrm{x}, \mathrm{y}, \mathrm{z}$ : coordinates of particle location, $\mathrm{m}$

$\mathrm{x}_{0} \quad$ : center of particle oscillation in $\mathrm{x}, \mathrm{m}$

$\mathrm{x}_{0}, \mathrm{y}_{0}, z_{0}:$ particle initial position

$Z \quad$ : electric mobility of particle, $\frac{\mathrm{m}^{2}}{\mathrm{Vs}}$

$\alpha \quad$ : intersection angle of laser beams, rad

$\delta \quad$ : fringe spacing, $\mathrm{m}$ 
: mean free path of medium gas, $m$

: wave length of laser beam, $\mathrm{m}$

: viscosity of medium gas, $\frac{\mathrm{kg}}{\mathrm{ms}}$

: gas density, $\frac{\mathrm{kg}}{\mathrm{m}^{3}}$

: particle density, $\frac{\mathrm{kg}}{\mathrm{m}^{3}}$

$\sigma=\mathrm{D}_{\mathrm{p}} \sqrt{\frac{\omega \rho_{\mathrm{g}}}{8 \mu}}$, dimensionless parameter in unsteady Stokes equation

$\tau \quad:$ relaxation time for particle motion, $\mathrm{s}$

$\phi \quad:$ phase lag of particle oscillation, deg

$\Omega \quad$ : angular frequency, $\frac{\mathrm{rad}}{\mathrm{s}}$

$\omega \quad$ : angular frequency of particle oscillation =fundamental angular frequency for the analysis, $\frac{\mathrm{rad}}{\mathrm{s}}$

\section{References}

1) Mazumder, M. K., et al. : Rev. Sci. Instrum., 48, 622 (1977) Renninger, R. G., et al. : ibid., 52, 242 (1981)

2 ) Yokoyama, T., et al. : Preprint of 25th Symposium on Powder Science, p82 (1987, Sapporo)

3 ) Sato, T., Nakajima, Y. : Trans. I. E. E. of Japan, 110, 473(1990)

4) Seely, S : "Electron Tube Circuit" 2nd ed., p603 (McGraw-hill Kougakusha, 1958 Tokyo)

5 ) Roberds, D. W. : Appl. Opt., 16, 1861(1977)

6) Moriguchi, S., et al. : "Suugaku Koushiki III", p211 (Iwanami Zensho, 1959 Tokyo) Gradshteyn, I. S., Ryzhik, I. M. : Table of Integrals, Series and Products, p974 (Academic Press, New York 1980)

7) Hino, M. : "Supekutoru Kaiseki (Spectrum Analysis)", p167 (Asakura Shoten, Tokyo 1977)

8 ) Sato, T. : Trans. I. E. E. of Japan, 107, 155 (1987)

9) Minami, S. ed. : "Hakei Deita Shori (Waveform Data Processing)", p40 (CQ Shuppansha, Tokyo 1986) 